\title{
Association between histone deacetylases and the loss of cochlear hair cells: Role of the former in noise-induced hearing loss
}

\author{
LI-TING WEN $^{1 *}$, JIE WANG $^{1,2^{*}}$, YE WANG $^{1}$ and FU-QUAN CHEN ${ }^{1}$ \\ ${ }^{1}$ Department of Otolaryngology-Head and Neck Surgery, Xijing Hospital, \\ Fourth Military Medical University, Xi'an, Shaanxi 710032; ${ }^{2}$ Department of Otolaryngology-Head \\ and Neck Surgery, Xi'an Children's Hospital, Xi'an, Shaanxi 710003, P.R. China
}

Received October 13, 2014; Accepted May 22, 2015

DOI: $10.3892 /$ ijmm.2015.2236

\begin{abstract}
Noise-induced hearing loss (NIHL) is one of the most frequent disabilities in industrialized countries. It has been demonstrated that hair cell loss in the auditory end organ may account for the majority of ear pathological conditions. Previous studies have indicated that histone deacetylases (HDACs) play an important role in neurodegenerative diseases, including hearing impairment, in older persons. Thus, we hypothesized that the inhibition of HDACs would prevent hair cell loss and, consequently, NIHL. In the present study, a CBA/J mouse model of NIHL was established. Following an injection with the HDAC inhibitor, suberoylanilide hydroxamic acid (SAHA), the expression levels of HDAC1, HDAC4 and acetyl-histone H3 (Lys9) (H3-AcK9) were measured. The number of hair cells was quantified and their morphology was observed. The results revealed that $1 \mathrm{~h}$ following exposure to $110 \mathrm{~dB}$ SPL broadband noise, there was a significant increase in HDAC1 and HDAC4 expression, and a marked decrease in the H3-AcK9 protein levels, as shown by western blot analysis. Pre-treatment with SAHA significantly inhibited these effects. Two weeks following exposure to noise, the mice exhibited significant hearing impairment and an obvious loss in the number of outer hair cells. An abnormal cell morphology with cilia damage was also observed. Pre-treatment with SAHA markedly attenuated these noise-induced effects. Taken together, the findings of our study suggest that HDAC expression is associated with outer hair cell function and plays a significant role in NIHL. Our data indicate that SAHA may be a potential therapeutic agent for the prevention of NIHL.
\end{abstract}

Correspondence to: Dr Fu-Quan Chen, Department of Otolaryngology-Head and Neck Surgery, Xijing Hospital, Fourth Military Medical University, 17 Changle Western Road, Xi'an, Shaanxi 710032, P.R. China

E-mail: fuquan_chen@126.com

${ }^{*}$ Contributed equally

Key words: histone deacetylases, noise-induced hearing loss, outer hair cells, suberoylanilide hydroxamic acid

\section{Introduction}

Noise-induced hearing loss (NIHL) is a major health issue in a number of industrialized countries, despite reduced occupational noise exposure, strict standards for hearing protection and extensive public health awareness campaigns (1). It has been demonstrated that the loss of hair cells in the auditory end organ accounts for the majority of ear pathological conditions (2). Cochlear hair cells include the inner hair cells and three rows of outer hair cells. Inner hair cells are the primary sensory cells that convert the mechanical acoustic input into receptor potential and the release of neurotransmitters, triggering action potentials in the auditory centers of the brain. The major function of outer hair cells is to enhance the performance of the cochlea, particularly at low sound-intensity levels (3).

Histone deacetylases (HDACs) are a class of enzymes that remove acetyl groups from the lysine residues of target proteins, promoting chromatin condensation and reduced transcription (4). Four classes of mammalian HDACs have been identified and classified based on sequence homology to the original yeast enzymes and domain organization $(5,6)$. HDAC1 is a member of class I HDACs, which are expressed in the majority of tissues and cell types and function as nuclear proteins and transcriptional repressors. HDAC4 belongs to class II HDACs, which are expressed in the heart, skeletal muscle and brain and function as a shuttle between the cytoplasm and the nucleus (7). The N-terminal tails of the core $\mathrm{H} 3$ histones contain specific amino acid sequences for acetylation, methylation, phosphorylation, ubiquitination and sumoylation (8), and H3K9 is a common modification site of acetylation (9). Previous studies have demonstrated that the treatment of mice with kanamycin decreases the acetylation of histone $\mathrm{H} 3$ in the nuclei of outer hair cells $(3,10)$.

Suberoylanilide hydroxamic acid (SAHA) is a commonly used HDAC inhibitor that effectively blocks all class I and class II HDACs. Studies have indicated that SAHA inhibits the growth of ovarian cancer cells, while inducing the expression of imprinted tumor suppressor genes (11-13). A previous study by our research group also demonstrated that the kanamycin-induced hypoacetylation of histones is associated with aminoglycoside-induced hair cell loss, and that the restoration of histone acetylation with HDAC inhibitors prevents damage to inner ear hair cells in vitro (3). However, whether there is an association between 
HDACs and NIHL, and the role of the HDAC inhibitor, SAHA, in preventing NIHL remains to be elucidated.

In the present study, hair cell death was assessed following the exposure of adult $\mathrm{CBA} / \mathrm{J}$ mice to broadband noise (BBN) to induce hearing loss. Following an injection of SAHA, the protein levels of HDAC1 and HDAC4 in the cochlear tissue and the levels of acetyl-histone H3 (Lys9) (H3-AcK9) in the outer hair cells were measured, the number of hair cells was quantified and their morphology was assessed, and comparisons were made between the mice injected with SAHA and the untreated mice in the control group.

\section{Materials and methods}

Antibodies and reagents. Unless otherwise stated, all materials were purchased from Gibco (Grand Island, NY, USA). Polyclonal rabbit antibodies against HDAC1 (Cat. no. 7872), HDAC4 (Cat. no. 11418) and H3-AcK9 (Cat. no. 33361), as well as the corresponding secondary antibodies were purchased from Santa Cruz Biotechnology (Santa Cruz, CA, USA). GAPDH antibodies (Cat. no. 367714) were obtained from Upstate Biotechnology, Inc. (Lake Placid, NY, USA). Alexa Fluor 594 secondary antibody (Cat. no. A24923), FITCphalloidin (Cat. no. F432) and Hoechst 33342 (Cat. no. H3570) were purchased from Molecular Probes (Eugene, OR, USA). SAHA was purchased from Cayman Chemical Co. (Ann Arbor, MI, USA). ECL Plus Western Blotting Detection reagents were purchased from GE Healthcare UK Ltd. (Little Chalfont, Buckinghamshire, UK).

Animals. Sexually mature (12 weeks old) male CBA/J mice were purchased from the Model Animal Research Center of Nanjing University (Nanjing, China). The animals were maintained on a 12-h light/12-h dark schedule and had free access to water and a standard mouse diet (Purina 5025) for 1 week before the commencement of the experiments. The animals were then randomly assigned to 3 experimental groups following weight stratification: i) the control group (G1), animals were placed in the noise exposure monitoring device, but were not exposed to noise; ii) the DMSO group (G2), animals were exposed to noise and received an intraperitoneal injection of DMSO (10\%) 3 days before they were exposed to noise; and iii) the SAHA group (G3), animals were exposed to noise and received an intraperitoneal injection of SAHA $(25 \mathrm{mg} / \mathrm{kg}) 3$ days before they were exposed to noise. Each group comprised 20 mice. All procedures were carried out in accordance with the Guide for the Care and Use of Laboratory Animals and were approved by the Medical Research and Ethics Committee (the Fourth Military Medical University).

Exposure to noise and auditory brainstem response $(A B R)$. The mice were kept in separate stainless-steel wire cages $(9 \times 9 \times 9 \mathrm{~cm})$ and the animals in the G2 and G3 groups were exposed to BBN with a frequency spectrum from 2 to $20 \mathrm{kHz}$ at a sound pressure level (SPL) of $110 \mathrm{~dB}$ for $2 \mathrm{~h}$ to induce a permanent threshold shift (PTS), while the mice in the G1 group were not exposed to noise in the same chamber. The experimental period lasted for 2 weeks.

Noise was created using a sound exposure chamber. Audio $\mathrm{CD}$ sound files were created and equalized with audio editing software (Adobe Systems Audition 3; Adobe Systems, Inc., San Jose, CA, USA). In order to ensure uniformity throughout the sound field, a sound level meter (model 1200; Quest Technologies Software, Aliso Viejo, CA, USA) was used to calibrate the sound levels at multiple locations within the sound chamber prior to and following the exposure of the mice to noise.

At the end of the experimental period, the mice were anesthetized with an intraperitoneal injection of xylazine $(20 \mathrm{mg} / \mathrm{kg})$ and ketamine $(100 \mathrm{mg} / \mathrm{kg})$, and were then placed in a sound isolation and electrically shielded booth (Acoustic Systems, Indianapolis, IN, USA). ABRs were measured at 4, 8, 12, 24, 32 and $48 \mathrm{kHz}$ using TDT system 3 hardware and SigGen/BioSig software (both from Tucker-Davis Technologies, Alachua, FL, USA). Thresholds were estimated between the lowest stimulus level at which a response was observed and the level without a response. All ABR measurements were conducted by the same experimenter blinded to the treatment conditions.

Western blot analysis. Cochlear tissue was homogenized and lysed with RIPA lysis buffer $(100 \mathrm{mM} \mathrm{NaCl}, 50 \mathrm{mM}$ Tris-HCl pH 7.5, 1\% Triton X-100, 1 mM EDTA, $10 \mathrm{mM}$ $\beta$-glycerophosphate and $2 \mathrm{mM}$ sodium vanadate and protease inhibitor). Protein concentrations were determined using the Micro BCA protein assay (Pierce, Rockford, IL, USA). A 40-mg sample of protein in each lane was separated by $10 \%$ SDS-PAGE and electroblotted onto polyvinylidenedifluoride (PVDF) membranes in a semi-dry trans-blot apparatus. Subsequently, the PVDF membranes were blocked by incubating them in $5 \%$ non-fat milk in TBST buffer at room temperature for $1 \mathrm{~h}$. The PVDF membranes were then incubated with anti-HDAC1, anti-HDAC4, anti-H3-AcK9, or anti-GAPDH antibodies for $1 \mathrm{~h}$ at room temperature. Following 3 washes in TBST buffer, HRP-conjugated secondary antibodies were introduced and ECL was used for detection.

Immunofluorescence staining and quantification of cell number. The mice were perfused through the heart with PBS and $4 \%$ paraformaldehyde (PFA) for 30 min under physiological pressure. The cochlear tissue was isolated and fixed with $4 \%$ paraformaldehyde for $12 \mathrm{~h}$ at $4^{\circ} \mathrm{C}$ and was then rinsed in PBS and decalcification buffer in a $4 \%$ solution of sodium EDTA (pH 7.4). The EDTA solution was changed daily for 3 days at $4^{\circ} \mathrm{C}$. Following decalcification, the cochleae were embedded in OCT-freezing medium and cut into $10-\mu \mathrm{m}$-thick serial sections. The corresponding sections were placed into $3 \%$ hydrogen peroxide for $2.5 \mathrm{~h}$ to quench endogenous peroxidase and were then incubated in a solution for blocking non-specific antibody binding overnight at $4^{\circ} \mathrm{C}$. Subsequently, the sections were processed with a primary antibody $(1: 100)$ for 4 days at $4^{\circ} \mathrm{C}$, washed in PBS and then incubated overnight at $4^{\circ} \mathrm{C}$ with Alexa Fluor 594-conjugated secondary antibodies. Nuclear material was detected by incubating the sections with Hoechst 33342 (Invitrogen, Carlsbad, CA, USA) for $30 \mathrm{~min}$ at room temperature. A sample size of 5 mice was used in each experimental group. Images were captured using a fluorescence microscope (BX50; Olympus, Essex, UK) using a DP Controller and DP Manager (Olympus, Essex, UK) software and a confocal microscope (FV-500; Olympus, Essex, UK). The number of cells expressing one or both markers of 
interest in each field was counted manually by 2 independent blinded observers using Adobe Photoshop. The percentage of hair cells was calculated as the average of 6 high-power fields of view normalized to the total number of cells per view.

Morphometric analysis. Hair cell morphometric changes were observed by phalloidin staining and scanning electron microscopy (SEM). The phalloidin-stained stereociliary bundles and circumferential F-actin rings on the cuticular plate of outer and inner hair cells allowed the determination of cells that were present or missing. The cochleae from the noise-exposed and unexposed groups were fixed and stained simultaneously with identical solutions and processed in parallel. FITC-conjugated phalloidin was used for labeling the hair cell structure to identify the comparable parts of hair cells for the capture of confocal images. Each condition was replicated in 4 different animals. SEM was performed after the cells were fixed in ice-cold $2.5 \%$ glutaraldehyde and $1 \%$ osmium tetroxide $2 \mathrm{~h}$ at $4^{\circ} \mathrm{C}$. Subsequently, the cells were dehydrated in an acetone gradient, then soaked and embedded in epoxy resin 618. Semithin sections $(1.0-\mu \mathrm{m}$-thick) were prepared after trimming and were located by light microscopy after staining. Ultrathin sections (70-nm-thick) were prepared and photographed using a scanning electron microscope (Olympus, Tokyo, Japan) following staining with uranyl acetate and uranium-lead acid.

Data analysis. Each experiment was repeated at least 3 times. The results are presented as the means \pm standard (SD). Statistical analyses were performed using a paired or unpaired Student's t-test for direct 2-group comparisons and the Tukey-Kramer post-hoc test after a one-way analysis of variance (ANOVA) F-test for multiple-group comparisons. A $\mathrm{P}<0.05$ was considered to indicate a statistically significant difference.

\section{Results}

Permanent NIHL in adult CBA/J mice. The exposure of 12-week-old male CBA/J mice to BBN at $110 \mathrm{~dB}$ SPL for $2 \mathrm{~h}$ resulted in PTS at 4, 8, 12, 24, 32 and $48 \mathrm{kHz}, 2$ weeks following exposure to noise (Fig. 1). Compared with the DMSO-injected group (G2), pre-treatment with the HDAC inhibitor, SAHA, significantly reduced PTS $(\mathrm{P}<0.05$ and $\mathrm{P}<0.01$; Fig. 1).

Traumatic noise exposure increases the espression of $\mathrm{HDACl}$ and HDAC4, but decreases H3-AcK9 levels. Western blot analysis revealed that the expression of HDAC1 and HDAC4 increased $1 \mathrm{~h}$ following exposure to noise and subsequently reached a plateau above the control levels (Fig. 2A). The quantification of the ratio of the protein band densities of HDAC1 revealed an approximately 2 -fold increase in protein expression compared with the control group, and an approximately 3 -fold increase was observed in the HDAC4 protein level (Fig. 2A). In contrast to the increase in HDAC1 and HDAC4 expression, the H3-AcK9 levels decreased $1 \mathrm{~h}$ following exposure to noise and showed lower band densities (Fig. 2B). The quantification of the band densities of $\mathrm{H} 3-\mathrm{AcK} 9$ to total $\mathrm{H} 3$ confirmed the significance of this decrease (Fig. 2B).

To address the issue of whether the expression of HDAC1, HDAC4 and H3-AcK9 is altered in the entire cochlea, an immunofluoresence assay was carried out. No apoptotic cells

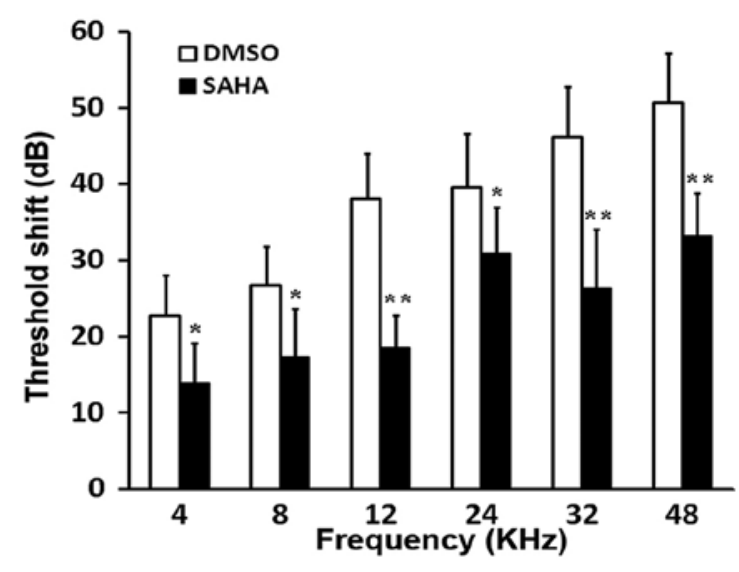

Figure 1. ABR threshold shifts. The bar graph represents permanent NIHL in 12-week-old male CBA/J mice 2 weeks following exposure to BBN at $110 \mathrm{~dB}$ SPL for $2 \mathrm{~h}$ each day. Permanent threshold shifts were greatest at high frequencies and decreased toward lower frequencies. Data are presented as the means $\pm \mathrm{SD} ; \mathrm{n}=5$ for each condition. ${ }^{*} \mathrm{P}<0.05$ and ${ }^{* *} \mathrm{P}<0.01$, compared with the DMSO-injected group. SAHA, suberoylanilide hydroxamic acid; ABR, auditory brainstem response; NIHL, noise-induced hearing loss; BBN, broadband noise; SPL, sound pressure level.

were observed in the normal controls. However, $1 \mathrm{~h}$ following exposure to noise, the outer hair cells in the basal segment showed typical cell death characteristics with the majority of cells exhibiting signs of apoptosis characterized by chromatin condensation (white arrows in Fig. 2C and D). This corresponded with an increase in HDAC4 levels and a decrease in H3-AcK9 levels.

Accoustic trauma increases hair cell loss, which is blocked following treatment with the HDAC inhibitor, SAHA. Seven days following the exposure of the mice to noise, the ratio of cochlear hair cell loss was calculated. Corresponding with the ABR functional measurements, large numbers of hair cells were lost in the basal and the middle regions of the epithelium. Outer hair cell loss began at a 5\% distance from the apex and increased to $60 \%$ cell loss the end of the epithelium. Inner hair cell loss also began at a 5\% distance from the apex and reached an average of $30 \%$ loss of hair cells at the end of the basal region (Fig. 3A).

Further analysis of hair cell loss following pre-treatment with the HDAC inhibitor, SAHA, revealed an interesting pattern. Compared with G2 (group injected with DMSO), the number of hair cells lost in the G3 (group injected with SAHA) was significantly decreased, particularly in the basal regions of the epithelium. Outer hair cell loss began at a $35 \%$ distance from the apex and increased to $40 \%$ cell loss the end of the epithelium, while inner hair cell loss began at an $80 \%$ distance from the apex and reached an average of $30 \%$ loss of hair cells at the end of the basal region (Fig. 3B), suggesting that SAHA partially inhibited the loss of hair cells induced by noise exposure, particularly outer hair cell loss.

The HDAC inhibitor, SAHA, protects outer hair cells against morphological changes induced by noise exposure. In order to investigate the morphological changes occurring in the ourter hair cells induced by noise exposure, and whether 

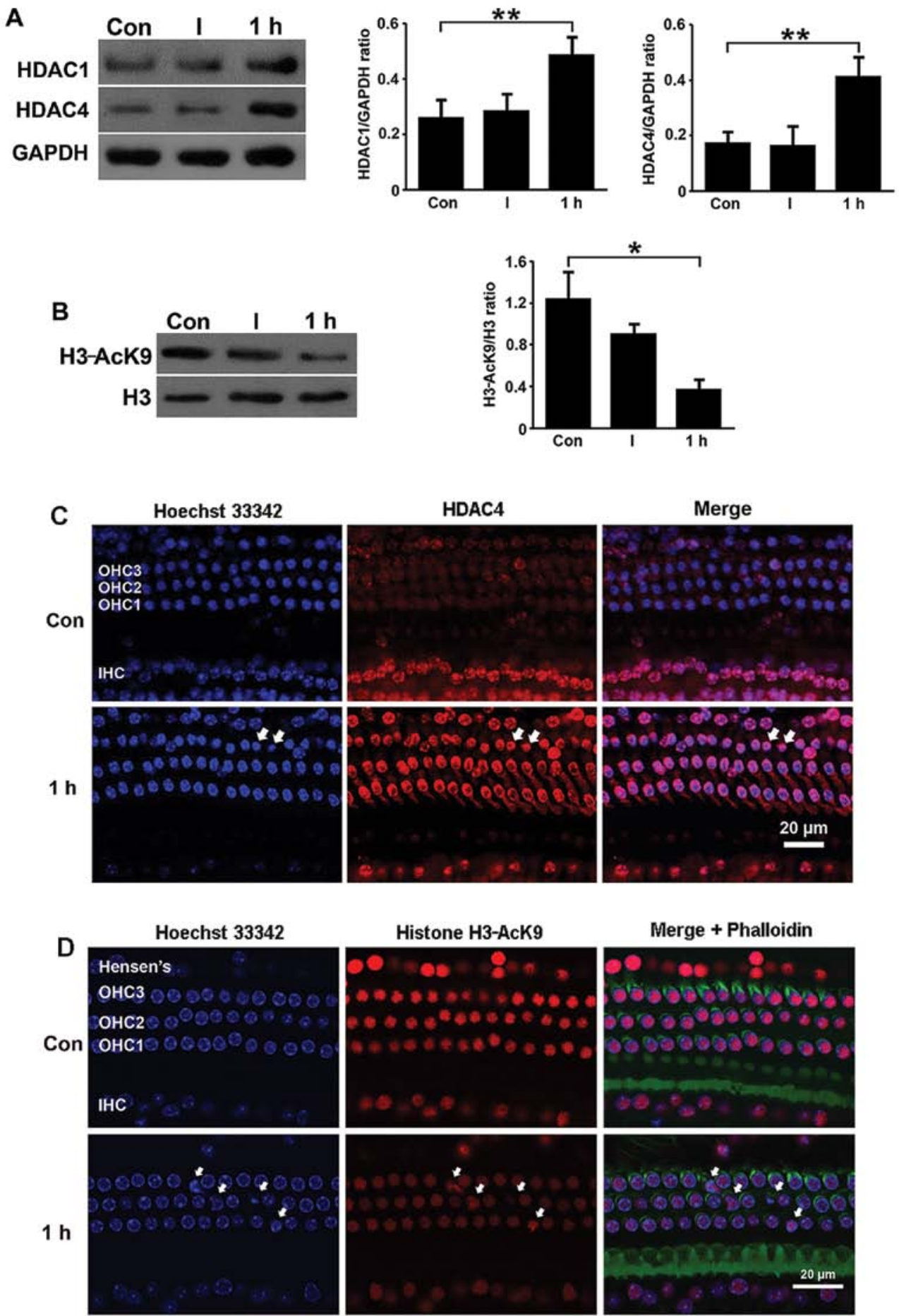

\section{Merge + Phalloidin}

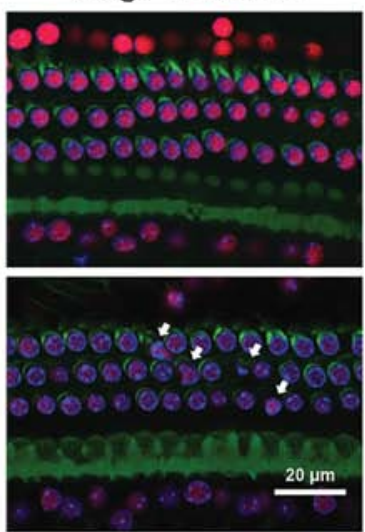

Figure 2. HDACs are transiently upregulated by traumatic noise. Western blot analysis detected HDAC-1 and HDAC-4 protein expression in the cochlear tissue. (A) One hour following the exposure of the mice to noise, there was an approximately 2-fold increase in the protein expression of HDAC1 and an approximately 3-fold increase in the HDAC4 protein levels compared with the control group. (B) Western blot analysis detected the H3-AcK9 protein levels in the cochlear tissue $1 \mathrm{~h}$ following exposure to noise and showed a significant decrease compared to pre-exposure. Con, control group; $\mathrm{I}, 0 \mathrm{~h}$ after exposure to noise; $1 \mathrm{~h}, 1 \mathrm{~h}$ after exposure to noise; ${ }^{*} \mathrm{P}<0.05$ and ${ }^{* *} \mathrm{P}<0.01$. (C) Immunofluorescence staining of the entire cochlea for HDAC4 (red) indicated a transient increase (white arrows) in the number of outer hair cells expressing HDAC4 $1 \mathrm{~h}$ after exposure to noise. (D) Immunofluorescence staining of the entire cochlea for H3-AcK9 (red) indicated a transient decrease (white arrows) in the number of outer hair cells expressing H3-AcK9 $1 \mathrm{~h}$ after exposure to noise. Nuclei are stained blue with Hoechst 33342 and Hensen's cells are identified by their position on the surface preparation and in comparison with remaining hair cells. Blue color represents Hoechst 33342 staining of the nuclei. The figure shows representative images from the basal segment ( $\mathrm{n}=3$ at each time point). Scale bar, $20 \mu \mathrm{m}$. HDAC, histone deacetylase; H3-AcK9, acetyl-histone $\mathrm{H} 3$ (Lys9); OHC1, OHC2, OHC3, outer hair cells of the first, second and third row, respectively; IHC, inner hair cells.

the HDAC inhibitor, SAHA, protects the cells against noise-induced damage, phalloidin staining (Fig. 4A) and SEM analysis (Fig. 4B) were carried out.
The results revealed that SAHA protected the outer hair cells against noise-induced damage. As shown in Fig. 4, the normal outer hair cells in the control group exhibited a normal 

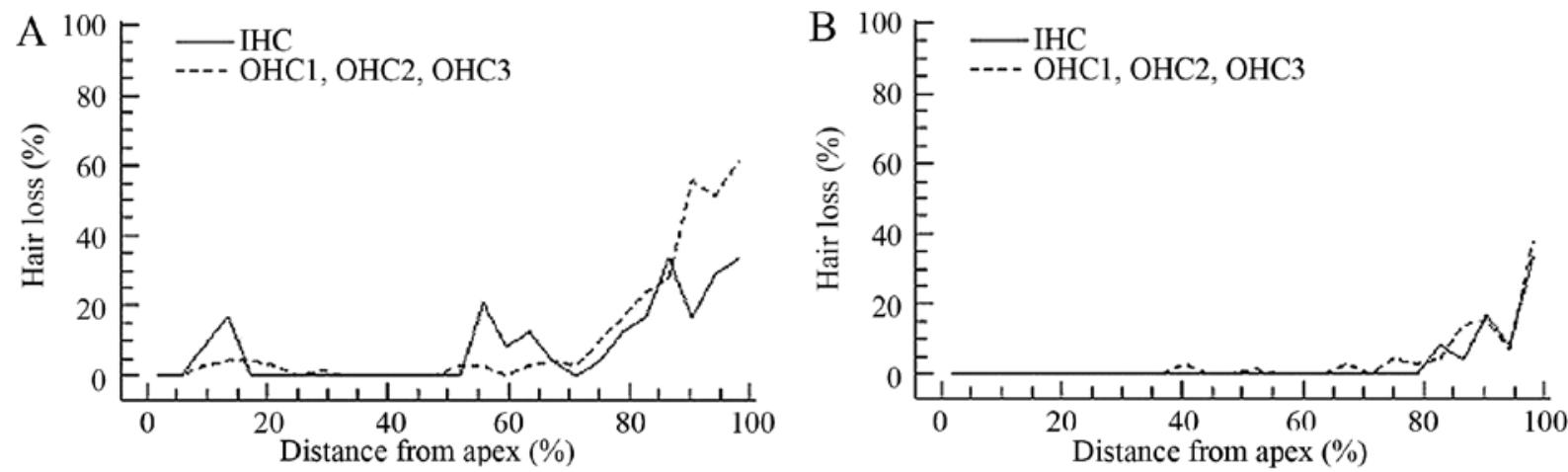

Figure 3. Quantification of hair cell loss. Both outer and inner hair cells were counted from the apex to the base along the entire length of the cochlear epithelium of the mice. (A) Both outer and inner hair cell loss increased along an apex-to-base gradient. (B) Following pre-treatment with the HDAC inhibitor, SAHA, both outer and inner hair cell loss decreased. OHC, outer hair cells; IHC, inner hair cells; HDAC, histone deacetylase; SAHA, suberoylanilide hydroxamic acid.
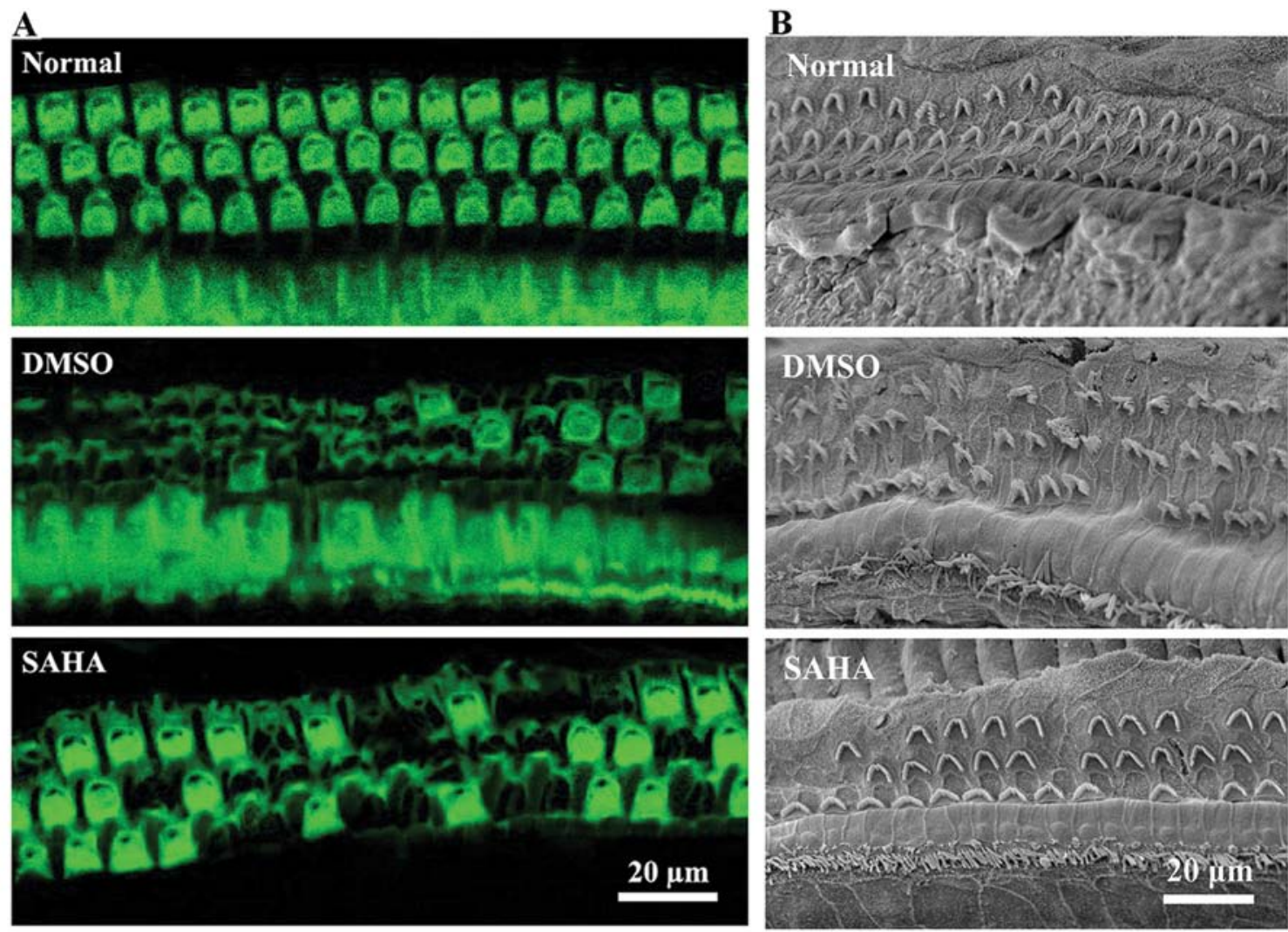

Figure 4. Noise-induced morphological changes in outer hair cells. Representative images of staining for F-actin with (A) phalloidin and (B) SEM images revealed obvious morphological changes with outer hair cell loss and cilia damage 2 weeks following exposure to noise, while pre-treatment with the HDAC inhibitor, SAHA, essentially doubled the number of surviving outer hair cells and attenuated cilia damage. Scales bar, $20 \mu \mathrm{m}$. HDAC, histone deacetylase; SAHA, suberoylanilide hydroxamic acid.

appearance. In G2 (DMSO treatment), 2 weeks following exposure to noise, cell morphology was significantly altered with outer hair cell loss and cilia damage. In G3 (SAHA treatment), in comparison to incubation with DMSO alone, essentially doubled the number of surviving outer hair cells and attenuated cilia damage. Thus, these results indicate that the HDAC inhibitor, SAHA protects outer hair cells against noise-induced morphological changes and prevents hair cell loss.

\section{Discussion}

NIHL is a type of permanent hearing impairment that results from prolonged exposure to high levels of noise (14). Hearing loss caused by recreational and occupational noise exposure is the second most common cause of sensorineural hearing loss following presbycusis (15). Histone acetylation and deacetylation, as important epigenetic modifications, are responsible for maintaining chromatin stability (16). An imbalance in 
histone acetylation has been implicated in a wide range of transcriptional dysfunctions, gene silencing and the development of neurodegenerative disorders (17-19). In the present study, we hypothesized that histone acetylation is involved in the death of inner ear sensory cells due to NIHL, and that the HDAC inhibitor, SAHA, may be used as a potential therapeutic agent to prevent hearing loss.

HDACs are a class of enzymes that remove acetyl groups from the lysine residues of target proteins, thus promoting chromatin condensation and reduced transcription (4). The overexpression or sustained HDAC activity has been reported in leukemia, lymphomas and other types of cancers, as well as in neurodegenerative disorders (20-22). Acetylated histones, such $\mathrm{H} 3$ and $\mathrm{H} 4$, serve as in vitro and in vivo markers for successful HDAC inhibition (23).

In this study, in order to determine the histone acetylation levels following exposure to BBN, an animal model of NIHL was established, and the protein levels of HDAC1, HDAC4 and $\mathrm{H} 3$-AcK 9 were measured by western blot analysis. The results revealed that, 2 weeks following the exposure of CBA/J mice to BBN, PTS at $4,8,12,24,32$ and $48 \mathrm{kHz}$ was evident in the ABR test, and a large number of hair cells was lost in the basal and middle regions of the epithelium, which presented as permanent NIHL in the adult $\mathrm{CBA} / \mathrm{J}$ mice. The HDAC1 and HDAC4 levels began to increase $1 \mathrm{~h}$ following exposure to noise and subsequently reached a plateau above the control levels, while the H3-AcK9 levels decreased at the same time point in the entire cochlea. This timing and location suggest that a change in histone acetylation may be one of the triggers to cell loss in the early stages of noise exposure.

HDAC inhibitors are a class of compounds that interfere with the function of histone deacetylase (24). Previous studies have investigated HDAC inhibitors as possible treatment strategies for cancer, neurodegenerative disorders and inflammatory diseases (25-28). It has been shown that the inhibition of HDAC1 and HDAC3 relieves Huntington's disease (HD)-like phenotypes in model systems and may be a target for human HD (29). In addition, as previously demonstrated, a novel class of pimelic $o$-aminobenzamide HDAC inhibitors may be used as therapeutic agents in neurodegenerative diseases, such as Friedreich's ataxia (30). Small-molecule inhibitors of class I, II and IV HDACs have been shown to exhibit anticancer activity with good safety profiles, particularly in patients with hematologic malignancies (31). Although the mechanisms of action of HDAC inhibitors remain unclear, they are emerging therapeutic agents that have been clinically validated in cancer patients with hematologic malignancies, including cutaneous T-cell lymphoma (32).

In the present study, pre-treatment with the HDAC inhibitor, SAHA, significantly reduced the threshold shifts 2 weeks following exposure to BBN, which suggests that SAHA may exert a protective effect against NIHL. To clarify the site and mechanism of action of SAHA, we investigated hair cell loss following pre-treatment with SAHA. The results revealed that following pre-treatment with SAHA, outer hair cell loss began at a $35 \%$ distance from the apex and increased to $40 \%$ cell loss at the end of the epithelium, while inner hair cell loss began at an $80 \%$ distance from the apex and reached an average of $30 \%$ loss of hair cells at the end of the basal region, which was significantly decreased compared with the control group.
These data suggest that SAHA partically inhibits noiseinduced hair cell loss, particularly outer hair cell loss. Further analysis of the morphological changes in noise-damaged outer hair cells by phalloidin staining and SEM revealed that SAHA protected the outer hair cells against noise-induced loss and morphological changes. This study lends strong support to the hypothesis that traumatic noise leads to the dysfunction of HDAC, causing cell loss and morphological changes in cochlear hair cells, particularly outer hair cells. Morevoer, the HDAC inhibitor, SAHA, significantly decreases NIHL, and may thus be a potential therapeutic strategy for the prevention of hearing loss. Several HDAC inhibitors have already been approved for clinical use $(33,34)$. Such inhibitors may benefit the large population of patients exposed to noise for long periods of time and are at risk of developing irreversible hearing and balance deficits.

\section{Acknowledgements}

The present study was supported by a grant from the National Natural Science Foundation of China (no. 81271069).

\section{References}

1. Sliwinska-Kowalska $\mathrm{M}$ and Davis A: Noise-induced hearing loss. Noise Health 14: 274-280, 2012.

2. Goodyear RJ, Jones SM, Sharifi L, Forge A and Richardson GP: Hair bundle defects and loss of function in the vestibular end organs of mice lacking the receptor-like inositol lipid phosphatase PTPRQ. J Neurosci 32: 2762-2772, 2012.

3. Chen FQ, Schacht J and Sha SH: Aminoglycoside-induced histone deacetylation and hair cell death in the mouse cochlea. J Neurochem 108: 1226-1236, 2009.

4. Shakespear MR, Halili MA, Irvine KM, Fairlie DP and Sweet MJ: Histone deacetylases as regulators of inflammation and immunity. Trends Immunol 32: 335-343, 2011.

5. Gao L, Cueto MA, Asselbergs F and Atadja P: Cloning and functional characterization of HDAC11, a novel member of the human histone deacetylase family. J Biol Chem 277: 25748-25755, 2002.

6. Morrison BE, Majdzadeh N, Zhang X, Lyles A, Bassel-Duby R, Olson EN and D'Mello SR: Neuroprotection by histone deacetylase-related protein. Mol Cell Biol 26: 3550-3564, 2006.

7. De Ruijter A, Van Gennip A, Caron H, Kemp S and van Kuilenburg A: Histone deacetylases (HDACs): characterization of the classical HDAC family. Biochem J 370: 737-749, 2003.

8. Sadri-Vakili G and Cha JH: Mechanisms of disease: histone modifications in Huntington's disease. Nat Clin Pract Neuro 2: 330-338, 2006.

9 Strasák L, Bártová E, Harnicarová A, Galiová G, Krejcí J and Kozubek S: H3K9 acetylation and radial chromatin positioning. J Cell Physiol 220: 91-101, 2009.

10. Jiang H, Sha SH and Schacht J: Kanamycin alters cytoplasmic and nuclear phosphoinositide signaling in the organ of Corti in vivo. J Neurochem 99: 269-276, 2006.

11. Hiebert SW and Stengel KR: Class I HDACs affect DNA replication, repair and chromatin structure: implications for cancer therapy. Antioxid Redox Signal: Jun 26, 2014 (Epub ahead of print).

12. Feng W, Zhang B, Cai D and Zou X: Therapeutic potential of histone deacetylase inhibitors in pancreatic cancer. Cancer Lett 347: 183-190, 2014

13. Rikiishi H: Autophagic and apoptotic effects of HDAC inhibitors on cancer cells. J Biomed Biotechnol 2011: Article ID 830260, 2011.

14. Rabinowitz PM: Noise-induced hearing loss. Am Fam Physician 61: 2759-2760, 2000.

15. Nair C: Noise-induced hearing loss. InnovAiT 7: 204-208, 2014

16. Kingston RE and Narlikar GJ: ATP-dependent remodeling and acetylation as regulators of chromatin fluidity. Genes Dev 13: 2339-2352, 1999.

17. Stilling RM and Fischer A: The role of histone acetylation in age-associated memory impairment and Alzheimer's disease. Neurobiol Learn Mem 96: 19-26, 2011. 
18. Song C, Kanthasamy A, Anantharam V, Sun F and Kanthasamy AG: Environmental neurotoxic pesticide increases histone acetylation to promote apoptosis in dopaminergic neuronal cells: relevance to epigenetic mechanisms of neurodegeneration. Mol Pharmacol 77: 621-632, 2010.

19. Gräff J and Tsai LH: Histone acetylation: molecular mnemonics on the chromatin. Nat Rev Neurosci 14: 97-111, 2013.

20. Bassett SA and Barnett MP: The role of dietary histone deacetylases (HDACs) inhibitors in health and disease. Nutrients 6 : 4273-4301, 2014.

21. Zwergel C, Valente S, Jacob C and Mai A: Emerging approaches for histone deacetylase inhibitor drug discovery. Expert Opin Drug Discov 10: 599-613, 2015.

22. Konsoula $Z$ and Barile FA: Epigenetic histone acetylation and deacetylation mechanisms in experimental models of neurodegenerative disorders. J Pharmacol Toxicol Methods 66: 215-220, 2012.

23. Vieyra D, Loewith R, Scott M, Bonnefin P, Boisvert FM, Cheema P, Pastyryeva S, Meijer M, Johnston RN and Bazett-Jones DP: Human ING1 proteins differentially regulate histone acetylation. J Biol Chem 277: 29832-29839, 2002.

24. Marks PA, Miller T and Richon VM: Histone deacetylases. Curr Opin Pharmacol 3: 344-351, 2003.

25. Chuang DM, Leng Y, Marinova Z, Kim HJ and Chiu CT: Multiple roles of HDAC inhibition in neurodegenerative conditions. Trends Neurosci 32: 591-601, 2009.

26. Halili MA, Andrews MR, Sweet MJ and Fairlie DP: Histone deacetylase inhibitors in inflammatory disease. Curr Top Med Chem 9: 309-319, 2009.
27. Huang L: Targeting histone deacetylases for the treatment of cancer and inflammatory diseases. J Cell Physiol 209: 611-616, 2006.

28. Dietz KC and Casaccia P: HDAC inhibitors and neurodegeneration: at the edge between protection and damage. Pharmacol Res 62: 11-17, 2010.

29. Jia H, Pallos J, Jacques V, Lau A, Tang B, Cooper A, Syed A, Purcell J, Chen Y, Sharma S, et al: Histone deacetylase (HDAC) inhibitors targeting HDAC3 and HDAC1 ameliorate polyglutamine-elicited phenotypes in model systems of Huntington's disease. Neurobiol Dis 46: 351-361, 2012.

30. Soragni E, Xu C, Cooper A, Plasterer HL, Rusche JR and Gottesfeld JM: Evaluation of histone deacetylase inhibitors as therapeutics for neurodegenerative diseases. Methods Mol Biol 793: 495-508, 2011.

31. Roger T, Lugrin J, Le Roy D, Goy G, Mombelli M, Koessler T, Ding XC, Chanson AL, Reymond, MK and Miconnet I: Histone deacetylase inhibitors impair innate immune responses to Toll-like receptor agonists and to infection. Blood 117: 1205-1217, 2011.

32. Kim HJ and Bae SC: Histone deacetylase inhibitors: molecular mechanisms of action and clinical trials as anti-cancer drugs. Am J Transl Res 3: 166-179, 2011.

33. Piekarz R and Bates S: A review of depsipeptide and other histone deacetylase inhibitors in clinical trials. Curr Pharm Des 10: 2289-2298, 2004.

34. Prince HM, Bishton MJ and Harrison SJ: Clinical studies of histone deacetylase inhibitors. Clin Cancer Res 15: 3958-3969, 2009. 